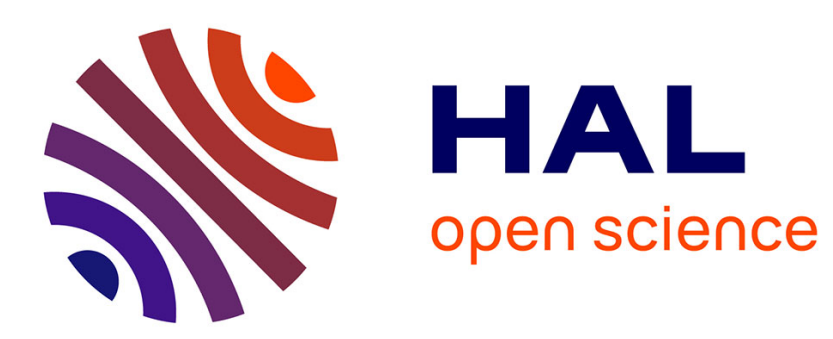

\title{
Réflexions sur le concept de qualité dans l'agro-alimentaire. L'exemple de la filière lait fromage dans les Alpes du Nord
}

Brigitte Dubeuf

\section{- To cite this version:}

Brigitte Dubeuf. Réflexions sur le concept de qualité dans l'agro-alimentaire. L'exemple de la filière lait fromage dans les Alpes du Nord. Productions Animales, 1992, 5 (2), pp.91-101. hal-00895965

\section{HAL Id: hal-00895965 https://hal.science/hal-00895965}

Submitted on 1 Jan 1992

HAL is a multi-disciplinary open access archive for the deposit and dissemination of scientific research documents, whether they are published or not. The documents may come from teaching and research institutions in France or abroad, or from public or private research centers.
L'archive ouverte pluridisciplinaire HAL, est destinée au dépôt et à la diffusion de documents scientifiques de niveau recherche, publiés ou non, émanant des établissements d'enseignement et de recherche français ou étrangers, des laboratoires publics ou privés. 
INRA Prod. Anim., $1992,5(2), 91-101$

\section{Brigitte DUBEUF}

INRA Unité de Recherches sur les Systèmes Agraires et Développement, Route de Saint-Cyr - 78026 Versailles Cedex
Réflexions sur le concept de qualité dans l'agro-alimentaire. L'exemple de la filière lait fromage dans les Alpes du Nord.

La qualité est un enjeu économique d'importance croissante sur les marchés des produits agro-alimentaires. Elle est également un enjeu central des relations entre les différents acteurs impliqués dans ces filières de production et dans l'aménagement d'un territoire. C'est pourquoi il nous est apparu intéressant de faire le point sur ce concept de qualité, en l'illustrant par l'exemple de la filière lait-fromage d'une zone de montagne, où son impact est vital.

\begin{abstract}
Depuis le début des années 80, la saturation des marchés internationaux et la limitation de l'offre des produits agricoles imposée par la Communauté Européenne ont profondément modifié le fonctionnement des marchés des produits agro-alimentaires. Dans de telles conditions, l'action sur les prix et les quantités n'assure pas à elle seule l'ajustement de l'offre et de la demande. La qualité des biens contribue également à réguler l'activité économique, comme l'énonce la théorie des conventions de l'économie de marché (EymardDuverney 1989).
\end{abstract}

On assiste en conséquence à un renforcement des règles propres à divers types de produits, telles que les appellations d'origine, les labels, les produits de l'agriculture biologique, l'appellation et la provenance "montagne", la certification de conformité. Parallèlement, pour les entreprises, "faire de la qualité" apparaît désormais comme "le prix à payer aujourd'hui pour être encore sur le marché de

\section{Résumé}

Le concept de qualité est soumis à des interprétations variées. La définition de la qualité des produits d'une filière n'est pas dictée uniquement par l'aval, mais résulte d'un processus complexe, dans lequel les relations entre agents sont déterminantes. La mise en place d'une politique de qualité nécessite une mobilisation collective à l'échelle d'une filière. Une démarche de modélisation est proposée pour construire des outils d'aide à la décision en matière de maitrise de la qualité. demain" (Valceschini 1991). Elles attaquent le marché en le segmentant pour mieux cibler la demande, et diversifient leurs produits pour $y$ répondre de manière plus spécifique. Elles cherchent également à garantir le niveau de qualité de leur production, grâce à la mise en place des systèmes d'assurance-qualité.

A la veille de l'ouverture du marché unique européen, nous nous proposons de faire le point sur le concept de "qualité" dans l'agroalimentaire, et sur ses difficultés d'application en nous appuyant sur l'exemple du secteur laitier, et plus particulièrement des filières lait-fromage dans les Alpes du Nord.

Le présent article, destiné à faire réfléchir sur le concept de qualité à partir d'une exploration de la littérature française consacrée à ce sujet, aborde d'une manière générale la notion de qualité, à travers la perception des économistes, des technologues, puis des différents acteurs de la filière agro-alimentaire : producteurs, entreprises et consommateurs. Il propose ensuite une application de ce concept de qualité à l'analyse des relations entre producteurs de lait et entreprises fromagères.

Un prochain article traitera des outils mis en place en vue du marché unique pour identifier et garantir la qualité des produits et de l'entreprise, et s'interrogera sur leur adaptation concrète aux besoins d'une filière telle que la filière lait-fromage des Alpes du Nord. 


\section{1 / Le concept de qualité des produits}

\section{1 / Une grille de lecture : les "modes de définition" de la qualité}

Dans le cadre du marché agro-alimentaire, la qualité des produits prend une place centrale, puisqu'elle participe activement à sa régulation. Comment aborder cette problématique de la qualité, dont chacun s'empare aujourd'hui ? Comment comprendre ce que les acteurs économiques mettent concrètement derrière ce terme ? Comment la Recherche peut-elle les aider à concevoir et à instrumenter des politiques de qualité adaptées à leurs besoins? Les réflexions actuelles sur les "modes de définition" de la qualité proposent, nous semble-t-il, un cadre d'analyse pertinent des discours et des pratiques qui se développent autour des politiques de qualité. C'est l'"économie des conventions" qui fournit le fil conducteur nécessaire, en s'appuyant sur l'étude des confrontations entre les agents économiques et qui amène à distinguer trois modes de définition de la qualité des produits (Sylvander 1991):

* Le premier mode de définition se réfère à des règles objectives, à des normes, qui conduisent à une standardisation des produits et des procédés de fabrication. On parle de "coordination industrielle" entre acteurs économiques.

* Le deuxième mode de définition est fondé sur la fidélité, l'estime, la confiance envers une personne, le fromager par exemple, et/ou un produit et sa marque. On parle de "coordination domestique".

* Le troisième mode de définition repose sur le prix du produit, établi selon le jeu de la concurrence, de l'offre et de la demande. On parle de "coordination marchande".

A côté de ces formes de coordination qui peuvent fonder des principes d'évaluation de la qualité des produits, on distingue enfin une forme de coordination fondée sur la solidarité collective, telle que l'on peut la concevoir par exemple entre une coopérative, ses producteurs et ses clients : il s'agit alors de "coordination civique" (Valceschini et Heintz 1991).

Selon leur logique de production, les entreprises aménagent des compromis entre les différentes formes de coordination. L'entreprise qui recherche l'amélioration des rendements et la réduction des coûts de production privilégiera la coordination industrielle. En revanche, celle qui souhaite garantir le maintien des modes de production et le renom de la marque développera davantage les relations domestiques (Boisard et Letablier 1987). Il en résulte que la définition de la qualité d'un produit dépend le plus souvent d'une combinaison entre les différentes formes de coordination (Lebail et Valceschini 1990).
Par exemple, si la qualité du reblochon fermier repose essentiellement sur des formes de coordination domestique (confiance envers le producteur et ses pratiques traditionnelles), et si celle du reblochon laitier se définit davantage à partir des formes de coordination industrielle (conformité au cahier des charges de l'appellation d'origine), la qualité de ces deux produits repose en réalité sur les trois formes de coordination :

- la demande est très saisonnière (coordination marchande),

- les caractéristiques des produits sont liées à un terroir et à une notion de tradition, que souligne le cahier des charges de l'appellation d'origine (coordination industrielle),

- l'image du fromage renvoie à celle de la montagne, qui traduit le naturel, le respect de l'environnement, etc. (coordination domestique).

La pluralité des logiques de production introduit la question du maintien de la cohérence d'une filière, ainsi que celle d'une entreprise (Eymard-Duverney 1989). Ceci permet de comprendre que les différentes formes de coordination qui cœxistent au sein d'une filière, comme celle du reblochon, peuvent amener des tensions et éprouver sa cohésion (selon Eymard-Duverney, la définition de la qualité constitue un élément d'autant plus sensible du paradigme du marché, que les acteurs économiques se trouvent dans une situation de plus grande incertitude quant au mode de définition de la qualité). Ainsi, dans une grande entreprise fromagère, l'automatisation de la production, souhaitée pour réduire les coûts, se heurte à la nécessité de conserver son caractère spécifique et son image à un fromage tel que le reblochon. Des tensions peuvent également se créer entre l'entreprise et ses producteurs, si leurs logiques de production sont peu compatibles. C'est pourquoi il semble important que les valeurs qui fondent la qualité des produits soient maintenues identiques tout au long d'une filière, depuis la production de la matière première jusqu'à la commercialisation du produit final (Boisard et Letablier 1987)

\section{2 / Les diverses définitions de la qualité}

En cette fin du XXè siècle, les objectifs en matière de produits agricoles sont devenus plus complexes et plus qualitatifs. Dans le domaine de la production laitière, la loi du 3 janvier 1969 et les décrets d'application du 16 novembre 1970 font obligation de payer le lait de vache selon sa composition et sa qualité bactériologique. Au cours des années 80 , la gestion de l'espace rural et la maîtrise des effluents d'élevage sont peu à peu pris en compte dans les objectifs officiels de l'agriculture. Les années 90 voient émerger la préoccupation du bien-être de l'animal.

La définition du produit agricole et plus particulièrement du produit alimentaire a 
également subi une évolution sensible, qui va globalement dans le même sens. Un produit alimentaire, un fromage par exemple, se définit à travers de multiples composantes, qui jouent toutes un rôle dans son éventuel succès commercial (encadré 1). Ce panorama montre l'importance et la complexité des paramètres de qualité qui vont entrer dans l'identification d'un produit.

\section{Composantes entrant dans la définition d'un produit}

(source : Buron 1990)

- L'objet (le produit en lui-même ; sa forme ; son emballage).

- Sa dénomination.

- Sa marque commerciale.

- Les composants du marketing : politique des prix ; politique de publicité ; politique de distribution.

- Son image pour le consommateur.

De nombreux auteurs ont étudié la sémantique de la qualité des produits. Ce concept a subi une évolution parallèle à celle des exigences du marché, que Buron (1990) résume en trois étapes :

1 - La qualité d'un produit se définit comme "l'ensemble des propriétés de l'aliment permettant une consommation satisfaisante" ;

2 - La qualité d'un produit se définit comme "une meilleure aptitude pour la consommation" ;

3 - La qualité d'un produit se définit comme "l'adaptation à son emploi" (Juran 1974).

La première définition fait référence aux normes dictées par les technologues et les scientifiques. Elle fige la qualité pour un produit donné. Dans ce sens, la qualité s'oppose à la non-qualité. Le désir du consommateur n'est, en pratique, pas pris en compte. Les composantes de la qualité qui sont prises en considération sont intrinsèques au produit et, pour la plupart, objectives et quantifiables (composition en éléments nutritifs, caractéristiques bactériologiques, etc.).

La troisième définition ne parle plus de "consommation satisfaisante", ni même d'"aptitude à la consommation", mais d'"adaptation" à un emploi non précisé. Elle exprime le fait réel que l'obtention d'un bon niveau de qualité, et la satisfaction du consommateur final, passe par la satisfaction de toutes les personnes ayant une relation avec le produit : les consommateurs bien sûr, mais aussi tous les intervenants des circuits de fabrication et de distribution, les producteurs et leurs conseillers techniques, etc. A l'échelle de l'entreprise, cette définition introduit le concept de "qualité totale" (Juran 1974), qui prend en compte l'ensemble des clients et des fournisseurs internes, et répond à l'idée que la qualité doit être élaborée pas à pas à l'intérieur de l'entreprise (Buron 1990).

Cette définition attire également l'attention sur l'importance de l'identification précise de l'emploi" des produits, en rapport avec le processus d'élaboration de la qualité : un produit doit être adapté aux spécifications imposées par son emploi. Ces spécifications précisent un certain nombre de conditions que le produit doit remplir pour satisfaire l'utilisateur. Elles fixent les objectifs qui permettent d'orienter les actions de l'entreprise dans une direction conforme à l'obtention de la qualité (Faillenet 1985, non publié). Les scientifiques, les techniciens, l'Administration, les professionnels et tous ceux qui interviennent dans l'élaboration de ces spécifications ont la charge de "traduire la voix du consommateur, mais sans introduire leur propre voix" (Buron 1990). La qualité du produit est évaluée grâce à un ensemble de critères qui doivent permettre de juger de sa conformité vis-à-vis des spécifications : la pertinence du choix de ces critères est donc essentielle pour la réussite d'une politique de qualité (figure 1).

La qualité attendue variant selon les utilisateurs et les différentes occasions de consommation, il est nécessaire de détecter avec précision les demandes du marché et de bien

Figure 1. Mécanisme de génération de la qualité d'un aliment (source : Buron 1990).

Critères de qualité
en relation avec les
emplois
Q
convenance entre les critères
et les spécifications imposées
par les emplois

\section{La qualité d'un produit prend en compte ses caractéristiques intrinsèques, par exemple sa composition, mais aussi un ensemble de critères traduisant son adéquation à la demande du consommateur.}


Tableau 1. Les différents aspects du concept de qualité d'un produit alimentaire. (source: Multon et Davenas 1985)

\section{1 - Qualité alimentaire :}

* qualité hygiénique : non toxicité

* qualité nutritionnelle : aptitude à bien nourrir

* qualité hédonique ( ou organoleptique, ou sensorielle) variable dans le temps et l'espace, et selon les individus.

\section{2 - Qualité psycho-sociale :}

"L'homme est un ommnivore qui se nourrit de viande, de végétaux et d'imaginaire" (Fischer 1979).

Ce type de qualité est lié à l'image que le consommateur se fait du produit. Cette image est notablement influencée par l'emballage du produit.

\section{3 - Qualité d'usage ou de service :}

* qualité technologique

* aptitude à la conservation :

- durée de vie après achat,

- durée de vie après ouverture de l'emballage

* commodité d'emploi du produit

* aspect économique : prix de vente

* aspects commerciaux : disponibilité, présentation, échange.

* aspect réglementaire : étiquetage.

cibler chaque segment de marché. En définitive, la qualité d'un produit dépend du degré d'adéquation entre les caractéristiques de qualité du produit conçu et mis sur le marché d'une part, celles du produit attendu par l'utilisateur d'autre part.

S'il existe actuellement un consensus des partenaires de l'agro-alimentaire pour définir le concept de qualité, conformément à la norme AFNOR (NF X 50.109), comme "l'en- semble des propriétés et caractéristiques d'un service ou d'un produit qui lui confère l'aptitude à satisfaire des besoins exprimés ou implicites", la qualité d'un produit alimentaire est toujours perçue à travers des objectifs concrets qu'il s'agit d'atteindre (tableau 1).

Cette perception du concept de qualité nous amène à la notion de qualité globale (Multon 1985), qui exprime l'idée selon laquelle la qualité d'un produit doit résulter d'une pondération entre les différents critères retenus dans sa définition. Elle pose également la question de l'objectivation de la qualité.

Les caractéristiques du lait conditionnent l'efficacité de l'ensemble du processus de transformation (rendement fromager, pourcentage de fromages déclassés...) qui aboutit à la production de fromage au lait cru entier, comme le reblochon. Diverses pratiques technologiques permettent certes de corriger certains défauts de la matière première, mais elles ont un coût non négligeable et une incidence sensible sur la qualité du produit fini. De plus, l'image des pratiques de production du lait est largement répercutée sur celle des fromages (respect de l'environnement par exemple). Les coopératives des Alpes du Nord qui fabriquent le reblochon ont donc des attentes particulières vis-à-vis de leurs producteurs. Ces attentes s'expriment à travers leur demande de qualité du lait, et l'attention qu'elles accordent à la stabilité de cette qualité au cours de l'année. Ceci est particulièrement évident dans le cas d'un fromage traditionnel de montagne, dont le positionnement commercial haut de gamme est très lié à l'image d'un fromage de terroir, au goût "typé", issu de systèmes de production respectueux des paysages alpins. Les critères de la qualité recherchée pour le lait cru produit

Pour les fromages traditionnels de montagne, le positionnement commercial haut de gamme est très lié à l'image d'un fromage de terroir, au goût "typé", issu de systèmes de production respectueux des paysages alpins.

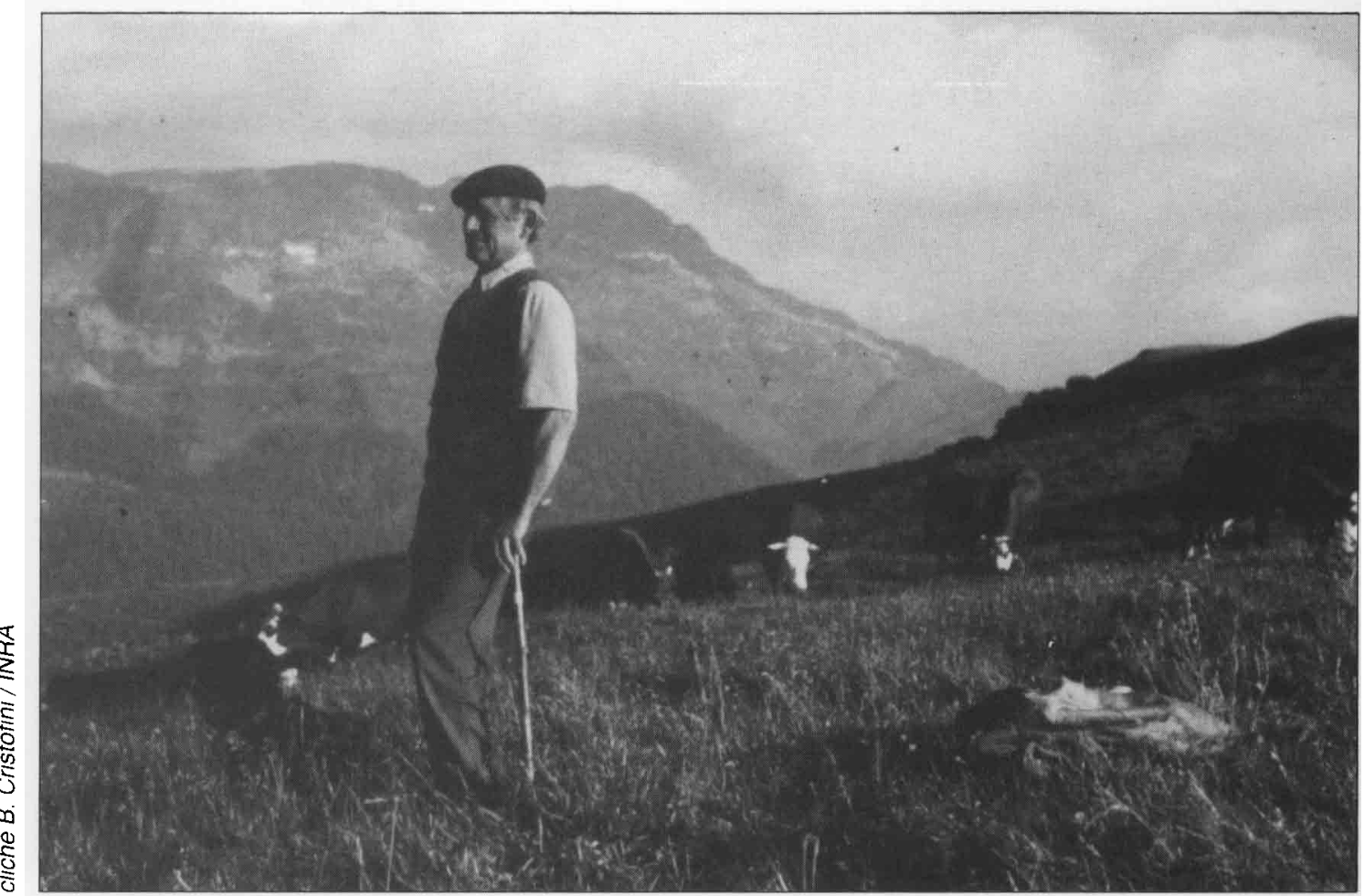


dans les Alpes du Nord pourraient être classés comme suit :

qualité bactériologique : nombre de germes, de cellules, absence de résidu d'antibiotiques,

- qualité technologique : taux protéique, nombre de spores butyriques ;

- qualité sensorielle : odeur, goût, couleur, texture :

- qualité d'usage : régularité des caractéristiques de la matière première, adaptation du volume de laits livrés au marché du fromage ;

- qualité psycho-sociale : conformité à l'image d'une production traditionnelle de fromage de terroir, respectueux de l'environnement et des traditions locales.

La décomposition de la qualité du produit en caractéristiques pouvant faire l'objet de mesures objectives permet d'harmoniser et de normaliser les critères de qualité ; elle tend vers la recherche d'une qualité standardisée du produit. La fonction d'usage, qui fait appel aux préférences diversifiées des consommateurs, renvoie davantage le jugement de la qualité du produit à un mode d'évaluation marchand, sanctionné par le jeu des prix (Eymard-Duverney 1989).

\section{2 / Le concept de qualité au sein d'une filière}

Les différents acteurs économiques d'une filière n'ont pas les mêmes besoins ni la même demande de qualité sur les produits de la filière. A un même niveau de la filière, les perceptions de la qualité peuvent en outre être très différentes.

\section{1 / Les producteurs et la qualité}

Reprenons l'exemple de la filière lait-fromage de montagne : tous les producteurs n'ont pas la même attitude vis-à-vis de la qualité du lait. Si la formule de fixation du prix du lait, qui tient compte de sa composition chimique et de critères bactériologiques, incite collectivement les producteurs à rechercher une qua. lité qui leur rapportera davantage, la gamme des laits livrés n'en reste pas moins très large, y compris sur les critères de qualité en question.

Cela est particulièrement net en zone de montagne, où les contraintes liées au milieu physique, aux structures d'exploitations et aux différents types de fromages fabriqués sont fortes, et où les objectifs des producteurs peuvent être très divers : réduction des coûts de production, aménagement du temps de travail en relation avec une double activité liée au tourisme, valorisation d'un patrimoine reçu en héritage, amélioration de la productivité du travail par l'augmentation du niveau de production du troupeau, etc.. Aussi, les techniques et les pratiques de production sont-elles variées. Certaines sont déterminantes pour la qualité des laits (Gilibert et al 1987). Mais leurs effets sur les performances laitières peuvent être différents d'un type d'exploitations à un autre (Coulon et al 1990).

Les exigences en matière d'approvisionnement pour la fabrication d'un fromage tel que le reblochon ne sont pas les mêmes pour une fromagerie industrielle que pour une fromagerie traditionnelle, car la perception de la qualité du lait est différente. Pour la première, la qualité du lait est plus à rechercher dans des critères bactériologiques et technologiques. Pour la seconde, ces critères de qualité sont également pris en considération, mais ils sont étendus à l'influence (encore mal connue) des caractéristiques du terroir, des pratiques traditionnelles et des moyens de production indigènes sur les aptitudes fromagères du lait qui découlent de sa composition chimique et de sa flore microbienne naturelle ainsi qu'à leur influence sur l'image associée au produit fini dans l'esprit du consommateur.

Ainsi, dans une filière donnée, les producteurs comme les transformateurs peuvent-ils avoir des perceptions et des attitudes très diverses vis-à-vis de la qualité du lait. Les relations entre tous ces agents économiques au sein de la filière intègrent nécessairement cette diversité d'approche, et la maîtrise collective de la qualité doit en tenir compte. Pour maintenir inchangées tout au long de la filière les valeurs qui fondent la qualité des produits, il est important, à cet égard, que les producteurs eux-mêmes aient une perception claire du positionnement du fromage sur le marché, et donc de ce qui fait sa qualité.

\section{2 / Les entreprises et la qualité}

Travailler pour la qualité d'un produit au sein d'une entreprise demande une prise de conscience à l'ensemble des acteurs de la "chaîne alimentaire" depuis le producteur de lait jusqu'au consommateur. Depuis le début des années 90 , quelques entreprises laitières ont fait de la politique de qualité un élément majeur de leur stratégie, et tentent d'orienter leur politique de marketing vers la satisfaction des besoins du consommateur. Elles sont encore très minoritaires.

De nombreux auteurs français (Multon et Davenas 1985), japonais et américains se sont penchés sur la politique de l'entreprise en matière de qualité. Cette politique se définit par un projet, autour duquel le groupe social représenté par l'entreprise va s'organiser et se structurer.

L'élaboration de la qualité d'un produit passe successivement dans l'entreprise par quatre étapes complémentaires, qui nécessitent de sa part quatre qualités distinctes (Bonnome 1990 non publié)

1 - la connaissance du besoin, qui nécessite d'être en permanence à l'écoute du consommateur (qualité d'écoute) ;

\section{Les différents intervenants d'une filière contribuent à l'élaboration de la qualité selon des conceptions et des critères parfois très divers - D'où la nécessité de définir collectivement un concept de qualité qui puisse traverser l'ensemble de la filière.}


Tableau 2. Le marché du fromage en l'an 2000. Source : GIRA (Richard 1990)

\section{1 - Les fromages du terroir :}

* Maintien du goût agricole (goût naturel à l'état brut, pouvant s'accompagner d'une certaine irrégularité).

* Orientation vers le goût "hypernaturel" (goût naturel et maîtrisé, mais varié), ce qui exige :

- la maîtrise de la qualité ;

- l'amélioration de la conservation.

\section{2 - Les fromages génériques :}

* Orientation vers les spécialités au goût industriel (goût maîtrisé jusqu'à la standardisation) par le développement d'une marque et d'un concept produit axé sur la "praticité d'utilisation".

* Orientation vers les fromages du terrain (le fromage dépasse le stade industriel pour accéder au statut hypernaturel).

\section{3 - Les spécialités :}

* Maintien du goût industriel ;

* Orientation vers le goût hypernaturel caractérisé par :

- une qualité irréprochable ;

- la maitrise des conditions de conservation ;

- une présentation esthétique.

2 - la pertinence du choix des spécifications, qui doivent être adaptées aux attentes identifiées, de manière à garantir au produit un degré de satisfaction donné du besoin (qualité de conception);

3 - l'adaptation de l'appareil de production à la fabrication programmée, qui permet de réaliser les produits conformément aux spécifications (qualité de réalisation);

4 - l'aptitude à détecter des "dérives de qualité", la mise en évidence de leurs causes et l'élaboration des actions correctives nécessaires pour obtenir la satisfaction visée des consommateurs envers les produits (qualité d'usage).

Ces étapes renvoient à des fonctions différentes de l'entreprise. Il faut donc, pour les atteindre, développer la motivation, la cohérence et la compétence de l'ensemble du personnel.

Dans le cas de l'approvisionnement des fromageries en lait, l'étape 1 consiste de leur part à positionner leur fromage par rapport à un créneau de marché bien identifié ; l'étape 2 à formuler clairement leurs exigences en matière de qualité du fromage et donc de qualité du lait (cahier des charges), les étapes 3 et 4 à s'informer sur la fréquence et l'importance des livraisons non conformes et à mettre en place les procédures susceptibles de les supprimer, et d'aboutir au strict respect du cahier des charges. Les deux principaux moyens d'action dont usent concrètement les entreprises laitières vis-à-vis de leurs fournisseurs sont d'une part l'information et le conseil délivrés par leurs services techniques, d'autre part la grille de fixation du prix du lait en fonction de sa qualité.
La réussite de la stratégie de qualité de l'entreprise repose sur une bonne connaissance de ses objectifs et de ses moyens (matières premières, process, matériels, hommes). Les "outils de la qualité" qui seront présentés dans un prochain article, contribuent à cette réussite.

L'entreprise doit donc s'efforcer de fournir des produits dont les caractéristiques sont définies en fonction de demandes spécifiques. D'après une étude du Groupe International sur les Recherches Alimentaires (GIRA) qui cherche à préciser les segments de marché du fromage de l'an 2000 (tableau 2), les petites entreprises laitières, pour acquérir un avantage concurrentiel face à la présence de plus en plus vive des mégacoopératives et des multinationales, devront privilégier deux axes de développement : l'innovation et le travail sur des produits de terroirs type AOC (Richard 1990).

\section{3 / Les consommateurs et la qualité}

Les études menées ces vingt dernières années sur l'analyse des déterminants de la consommation alimentaire montrent que si le statut social et le niveau des ressources restent des critères discriminants pour la différenciation des consommations (Bourdieu 1979, Grignon et Grignon 1980), "la structure des emplois du temps des ménages" (Herpin 1980), donc le mode de vie et le manque de temps (Sylvander 1988), influent fortement sur les attitudes des consommateurs vis-à-vis des caractéristiques d'usage des produits.

Parallèlement, les consommateurs recherchent une plus grande sécurité alimentaire. Le "poulet aux hormones" a été en quelque sorte un révélateur. La part croissante des produits industriels ne fait qu'amplifier l'incertitude des consommateurs sur les qualités des produits alimentaires. Cette incertitude se concrétise par la recherche de produits pour lesquels le recours à une pratique traditionnelle et/ou artisanale pourrait être garantie et par l'intérêt accru pour les produits dits "naturels", "frais" et "biologiques" (Sylvander et al 1986).

De fait, l'étude précitée du GIRA sur le marché du fromage en l'an 2000 fait ressortir des segments de marché en plein essor (Richard 1990) :

- un marché basé sur la "praticité d'utilisation" : le consommateur attend des produits adaptés aux différentes occasions de consommation, par exemple les fromages emballés en parts individuelles.

- un marché développant la composante "naturelle" : Dans cette optique, les fromages traditionnels, les fromages frais et les fromages naturels (ni allégés, ni enrichis) ont de bonnes perspectives de développement.

- un marché des produits dits "nutritionnels" ou diététiques (allégés en matières grasses, appauvris en cholestérol, enrichis en vitamines, etc.). 
Si les consommateurs se trouvent dans l'incertitude face à la qualité des produits, ils le sont également face aux facteurs de production de cette qualité. L'exemple connu du Poulet Label le démontre : le consommateur définit sa qualité supérieure par une bonne adhérence de la viande sur la carcasse et l'explique par la spécificité de l'alimentation (Sylvander 1991), alors que ce critère de qualité est fortement corrélé avec l'âge à l'abattage (Touraille et al 1981).

La qualité des produits et le respect de l'environnement sont des préoccupations qui se sont affirmées au fil de ces vingt dernières années. Aussi sont-ils souvent associés dans l'esprit du public comme dans de nombreux discours politiques. Or, ce sont deux problèmes très différents, qui peuvent difficilement être résolus simultanément, d'une part à cause de leur spécificité respective, d'autre part en raison de leur statut économique différent (usage privatif pour le premier, collectif pour le second), ainsi que le relève Thiébaut (1991). Les produits d'Appellation d'Origine Contrôlée jouent certes sur la relation qualitéenvironnement. Ainsi certains fromages appuient-ils leur publicité sur la qualité d'un paysage préservé. Mais si la nature du terroir contribue bien à la qualité des produits, l'impact sur l'environnement des systèmes de production qui élaborent ces produits est rarement envisagé.

Des catégories de consommateurs recherchent même des produits élaborés dans le respect de certaines valeurs sociales, qui contribuent au maintien de la qualité de l'environnement, et cela indépendamment de la qualité intrinsèque du produit (Thiébaut 1991). Mais la plupart des demandes des consommateurs sont satisfaites par des produits qui ont en réalité, directement ou non, une incidence de plus en plus négative sur l'environnement (demande d'emballage, de réfrigération...). On peut donc penser que le marché des produits de qualité "respectueux de l'environnement" ne se développera à terme que si s'instaure véritablement une convergence entre d'une part la qualité des produits et d'autre part l'incidence sur l'environnement des pratiques liées à leur production.

\section{3 / Le concept de qualité des produits agricoles}

\section{1 / Qualité "interne" et qualité "externe"}

Plus encore qu'un produit alimentaire, le produit agricole destiné à la transformation est souvent perçu à travers son système de production. Ceci est lié en partie à un élargissement de la conception de la qualité chez les participants de la filière. Aussi, pour des produits agricoles, ce concept comporte généralement deux volets (Demeyer 1991, Thiébaut 1991) :
- la qualité "interne", ou qualité directement liée au produit ;

- la qualité "externe", ou qualité "indirecte", liée à son système de production. Dans le cas d'une demande de produits "respectueux de l'environnement", la qualité "externe", définie comme "le respect par les processus de production d'un certain nombre de valeurs" (Thiébaut 1991), devient fondamentale. Le Beaufort, par exemple, véhicule l'image d'une montagne humanisée et s'accomoderait mal d'une montagne en friche.

Aux yeux des entreprises laitières, l'organisation de la production et de la collecte entrent dans la définition de la qualité du lait. La gestion de la qualité du lait consiste en effet à maîtriser non seulement des critères bactériologiques, technologiques, mais aussi la variation au cours de l'année du volume livré sur l'ensemble de la zone de collecte. Le fait qu'il soit possible de payer au même prix, dans la même entreprise, deux laits de qualité bactériologique et technologique très différente (tableau 3), ou à l'inverse que le prix d'un lait de qualité constante varie selon la saison montre clairement que le concept de qualité du lait inclut, pour les entreprises, sa qualité externe.

\section{2 / Analyse de la qualité d'un produit agricole : le lait}

D'un point de vue technique ou économique, la qualité du lait, telle qu'elle est évaluée par les critères définis plus haut, peut être consi-

Tableau 3. Mode de calcul du prix du kg de lait destiné à la transformation fromagère dans une coopérative de Haute-Savoie en zone Reblochon (1989). Comparaison de deux laits de caractéristiques très différentes.

\begin{tabular}{|c|c|c|}
\hline \multirow[t]{2}{*}{ Critères de qualité } & \multicolumn{2}{|c|}{ incidence sur le prix $(\mathrm{F} / \mathrm{kg})$} \\
\hline & LAIT A & LAIT B \\
\hline Période de production & février : $+0,10$ & mai : $-0,14$ \\
\hline Nombre de germes & $\begin{array}{l}>300000:-0,15 \\
\quad(\text { classe } \mathrm{C})\end{array}$ & $\begin{array}{c}<100000:+0,02 \\
(\text { classe A) }\end{array}$ \\
\hline Nombre de cellules & $\begin{array}{l}>300000: 0 \\
\quad(\text { classe B })\end{array}$ & $\begin{array}{c}<200000:+0,03 \\
(\text { classe A) }\end{array}$ \\
\hline Nombre de spores butyriques & $250: 0$ & $<180:+0,02$ \\
\hline Taux protéique $(\mathrm{g} / \mathrm{kg})$ & $28:-0,123$ & $31,5: 0$ \\
\hline Taux butyreux $(\mathrm{g} / \mathrm{kg})$ & $36,5: 0$ & $36,5: 0$ \\
\hline $\begin{array}{l}\text { Valeur totale du facteur } \\
\text { correctif lié à la coopérative }\end{array}$ & $+0,085$ & $+0,085$ \\
\hline Prix de base $=f(M P N)^{*}$ & 2,349 & 2,252 \\
\hline $\begin{array}{l}\text { Prix du kg de lait } \\
\text { payé au producteur }\end{array}$ & $2,261 \mathrm{~F}$ & $2,267 \mathrm{~F}$ \\
\hline
\end{tabular}

* MPN= Moyenne Pondérée Nationale de l'Emmental Est Central (voir encadré 2).

\section{Le prix du lait destiné à la fabrication fromagère tient compte non seulement des caractéristiques technologiques et bactériologiques mais aussi de critères tels que le mois de production.}


dérée comme une performance de production, dans la mesure où ce terme désigne précisément tout indicateur ou combinaison d'indicateurs permettant de juger de la qualité d'un animal, d'un système d'élevage ou d'un processus de production (Landais 1987). A la différence d'autres performances de production, la qualité du lait apparaît certes complexe, aussi bien par son mode de définition que par son objectivation, et la part de subjectivité dans la définition de la qualité peut être très importante. Néanmoins, cette assimilation de la qualité à une performance particulière a l'intérêt de situer le problème de sa maîtrise dans une problématique plus générale, qui est celle de la "modélisation de l'élaboration des performances" (figure 2). L'objectif de ce ce type de démarche consiste à fournir aux acteurs concernés des outils d'aide à la décision, principalement pour ce qui concerne les choix stratégiques, qui sont au centre du débat sur les politiques de qualité.

Cette problématique intègre les questions relatives au mode de définition et à l'objectivation de la qualité. Son mode de définition relève des systèmes de relations entre producteurs et organismes collecteurs de lait (Boisard et Letablier 1987, Eymard-Duverney

Figure 2. Processus d'élaboration de la qualité du lait destiné à la fabrication fromagère.

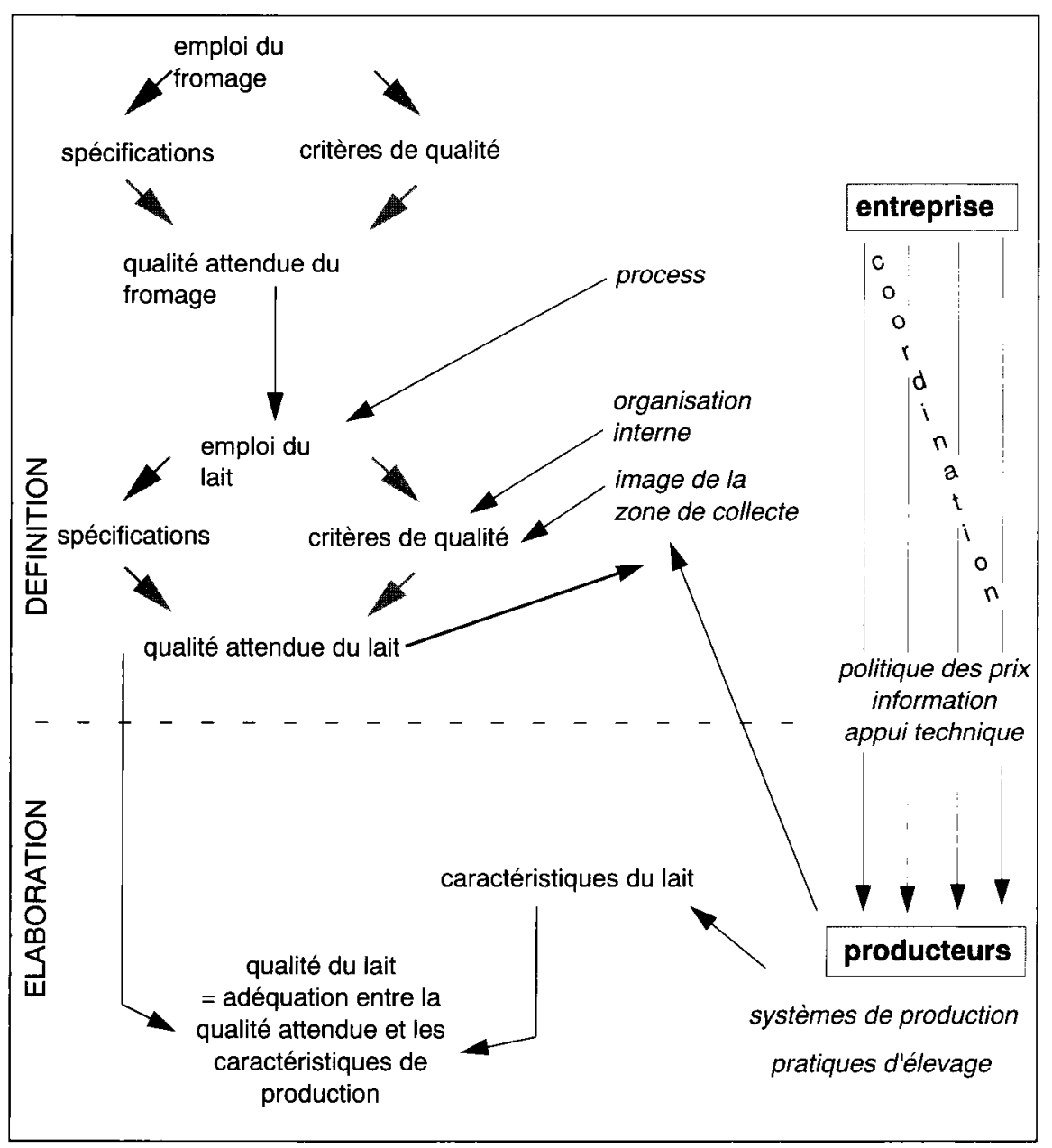

\section{Relations entre producteurs agricoles et entreprises laitières dans les Alpes du Nord : la fixation du prix du lait.}

Pour préserver le consommateur, l'Administration impose de strictes règles hygiéniques et sanitaires, qui normalisent la définition et les modes d'évaluation des caractéristiques bactériologiques (germes, cellules, résidus d'antibiotiques) qui ont une répercussion directe sur la qualité du lait. Les entreprises réagissent en instaurant un paiement du lait différent selon la classe bactériologique. De même, pour satisfaire aux exigences de la transformation fromagère, l'entreprise peut être amenée à normaliser des critères technologiques (taux protéique, spores butyriques, lipolyse), qui vont alors intervenir dans les modalités d'évaluation du lait. Mais certains paramètres entrant dans la fixation du prix du lait, et surtout du "prix de base", peuvent échapper totalement à l'entreprise.

Dans l'Isère, la fixation du prix de base est liée à un consensus entre entreprises. Elle dépend en Savoie comme en Haute-Savoie de la moyenne pondérée nationale de l'Emmental Est Central. Mais en HauteSavoie elle est soumise aussi au jeu de la concurrence entre l'offre et la demande de lait, et diffère selon la zone d'appellation (Reblochon ou Emmental). Enfin, dans les coopératives en gestion directe de Savoie, elle fluctue avec le marché du fromage.

1989, Valceschini 1991). La question ne se pose donc pas sous une forme unique et générale pour une filière de produits donnée, mais sous des formes variées selon la stratégie des entreprises et la "panoplie" de producteurs de chacune d'entre elles. Ce tissu de relations entre producteurs et entreprises repose sur des confédérations sociales et économiques, parfois très localisées (Caneill et Capillon 1990). La gestion des quotas laitiers, les modalités d'évaluation et de paiement du lait, le niveau local de la concurrence, les rapports de force établis entre producteurs, transformateurs et affineurs, le réseau relationnel (agents de Développement, agents commerciaux), etc., en forment la trame. Tous ces éléments exercent une influence sur la gestion de la qualité. La diversité des méthodes de fixation du prix du lait dans les Alpes du Nord illustre bien la complexité de ce type de problème (encadrés 2 à 4 ).

Il est clair que la qualité du lait recherchée par l'entreprise est en partie dictée par l'aval :

- d'une part, la qualité intrinsèque d'un fromage est en étroite relation avec celle du lait, et sa qualité "externe" avec l'image des systèmes de production et des paysages agraires ;

- d'autre part, le caractère saisonnier des livraisons de lait doit être pris en compte non seulement pour des raisons techniques (il 
peut pénaliser la fabrication de certains fromages), mais aussi parce que le planning de production doit s'adapter à la saisonnalité de la demande. C'est pourquoi le prix du lait, à qualité intrinsèque constante, varie largement au cours de l'année, avec une amplitude différente selon le contexte (figure 3).

La qualité de la matière première souhaitée par l'entreprise dépend également de son process (techniques de production et contraintes de fabrication) et probablement, dans une certaine mesure, de la perception qu'elle a de ses producteurs-fournisseurs et de leur offre de qualité, comme l'ont montré Lebail et Valceschini (1990) dans le secteur du blé dur destiné à la semoulerie-patisserie. La représentation que se fait l'entreprise de sa zone de collecte, du point de vue de la géographie agraire mais aussi à travers la diversité perçue des systèmes de production mis en œuvre par ses fournisseurs (en termes d'atouts et de contraintes vis-à-vis de la qualité du produit livré), peut donc exercer une influence sur la définition de la qualité qu'elle recherche!

La qualité du lait recherchée par l'entreprise se traduit par le choix des critères de qualité, qui sont définis, comme nous l'avons $\mathrm{vu}$, en conformité avec les spécifications. Dans le cas d'un fromage d'Appellation d'Origine Contrôlée (AOC) fabriqué au lait cru, ces spécifications sont imposées par le décret relatif à l'AOC, par les dispositions réglementaires concernant les laits crus (décret du 21 mai 1955 ) et la liste des critères microbiologiques auxquels doit satisfaire le lait destiné à la transformation (arrêté du 17 septembre 1984).

\section{Relations entre producteurs agricoles et entreprises laitières dans les Alpes du Nord : la gestion des quotas laitiers.} (source : Buisson et Guglielmi 1990).

En Savoie, les quotas laitiers sont gérés par un GIE départemental, qui est l'unique acheteur ONILAIT. Cette situation a permis à la zone de production de Beaufort de continuer à progresser en lait. Dans cette région, les fruitières fabriquent un fromage de qualité, dont la demande est soutenue. La forte croissance de la production laitière est assurée par des petits livreurs. Pour maintenir cette croissance, les fruitières n'hésitent pas à soutenir, voire à pousser leurs producteurs. En revanche, dans d'autres zones du département, certaines coopératives, par crainte des pénalités, ont freiné leurs producteurs, entraînant même un renversement de situation se traduisant par une diminution trop importante de leur livraison de lait! En Haute-Savoie, un système de prêt de quotas assure à tous les producteurs ayant besoin de croissance les droits à produire nécessaires. En pratique, il a surtout bénéficié aux gros livreurs.
Figure 3. Evolution saisonnière du prix d'un $\mathrm{kg}$ de lait de composition constante * au cours de l'année 1990. Comparaison entre 2 coopératives. (* TP $=31,5 \mathrm{~g} / \mathrm{kg}$, $T B=36,5 \mathrm{~g} / \mathrm{kg},<100000$ germes $/ \mathrm{ml},<200000$ cellules $/ \mathrm{ml},<180$ spores butyriques/l).

\section{$\mathrm{F} / \mathrm{kg}$}

2,6 Coopérative de Haute-Savoie en zone Reblochon

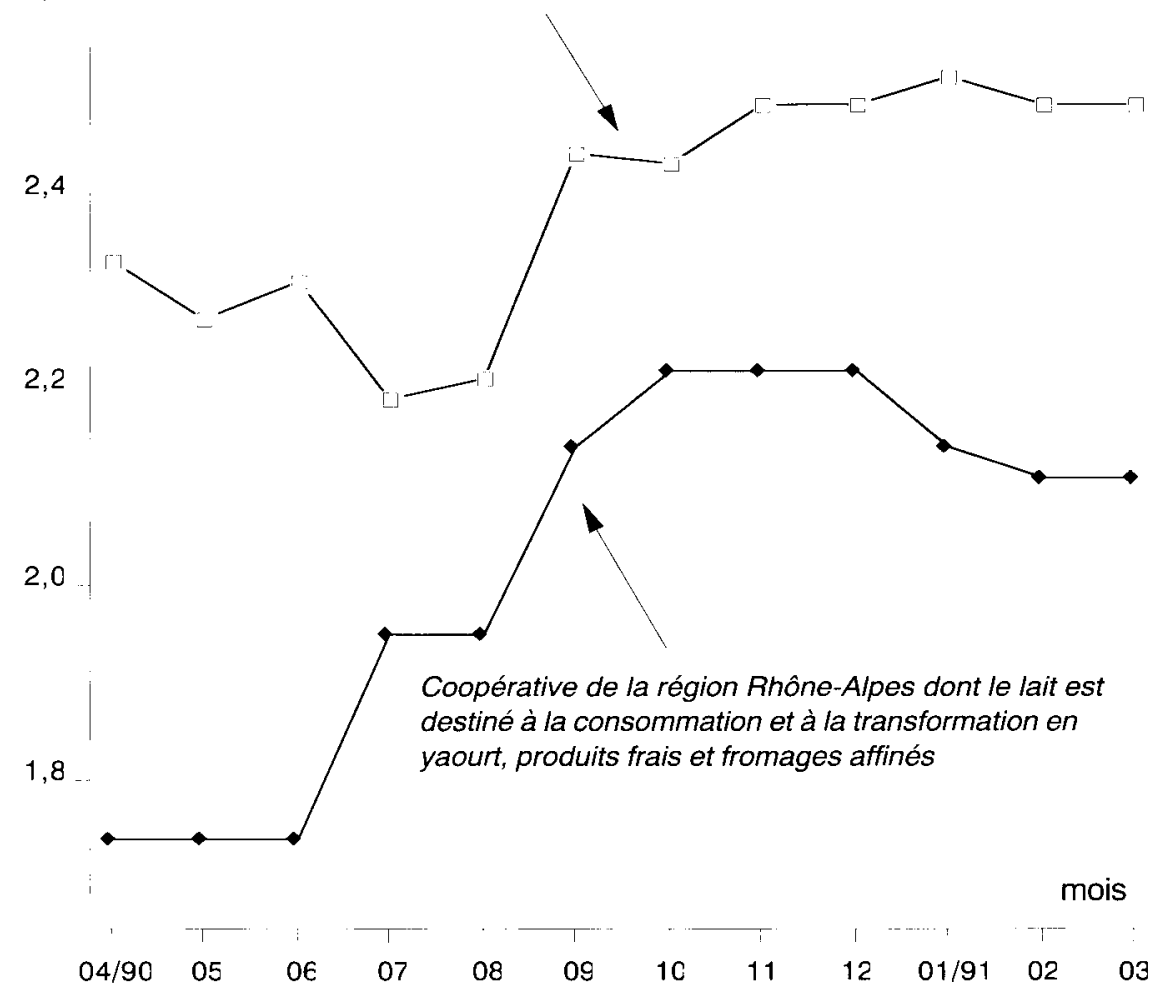

La modélisation du processus d'élaboration d'une performance, en vue d'une meilleure maîtrise de son obtention, passe par une phase de décomposition de la performance globale en composantes, puis par une phase d'analyse des facteurs de variation de chaque composante et des pratiques par lesquelles les opérateurs peuvent intervenir pour modifier dans un sens ou dans un autre le niveau de ces composantes. A l'échelle d'un système complexe, cette modélisation suppose que l'on connaisse tous les phénomènes biotechniques mis en jeu, mais également la manière spécifique dont ils se combinent, en raison des multiples interactions possibles (Landais 1987).

Dans la perspective d'une meilleure mâ̂trise de la qualité dans une filière fromagère de montagne, considérée comme un système complexe piloté par les acteurs de ses différents niveaux d'organisation, le "processus d'élaboration de la qualité du lait" pourrait ainsi être étudié, en suivant cette démarche, à la lumière du processus global de la filière (production-transformation-commercialisation-consommation) et de l'analyse des relations entre ses divers participants. L'une des retombées attendues d'une telle étude pourrait consister à amener les entreprises laitières à améliorer la représentation qu'elles se font de leur bassin de collecte et à adopter des politiques de qualité plus diversifiées, plus 


\section{Relations entre producteurs agricoles et entreprises laitières dans les Alpes du Nord : le partage de la valeur ajoutée.}

Dans certaines filières fromagères protégées par une appellation d'origine, les acteurs économiques ont mis en place des stratégies de différenciation des produits et des mécanismes puissants de protection du marché. Ces filières ont bénéficié ainsi d'une rente de monopole, dont la redistribution a été plus ou moins bénéfique aux producteurs selon le rapport de force entre les producteurs, les transformateurs et les affineurs (PerrierCornet 1990).
Le Beaufort a su générer, par un positionnement haut de gamme, une forte valeur ajoutée, qui profite largement aux producteurs organisés en fruitières. Le cas du reblochon est plus complexe, en partie du fait de la segmentation de son marché. La filière reblochon laitier bénéficie également d'une valeur ajoutée élevée, qui a été accaparée en grande partie par les transformateurs. Toutefois, le prix payé aux producteurs de lait, quoique plus fluctuant que celui de la zone Beaufort, est relativement élevé, grâce à la création des zones d'appellation. Dans le segment de filière occupé par le reblochon fermier, les producteurs-fromagers profitent plus complètement de la forte valeur ajoutée du produit.

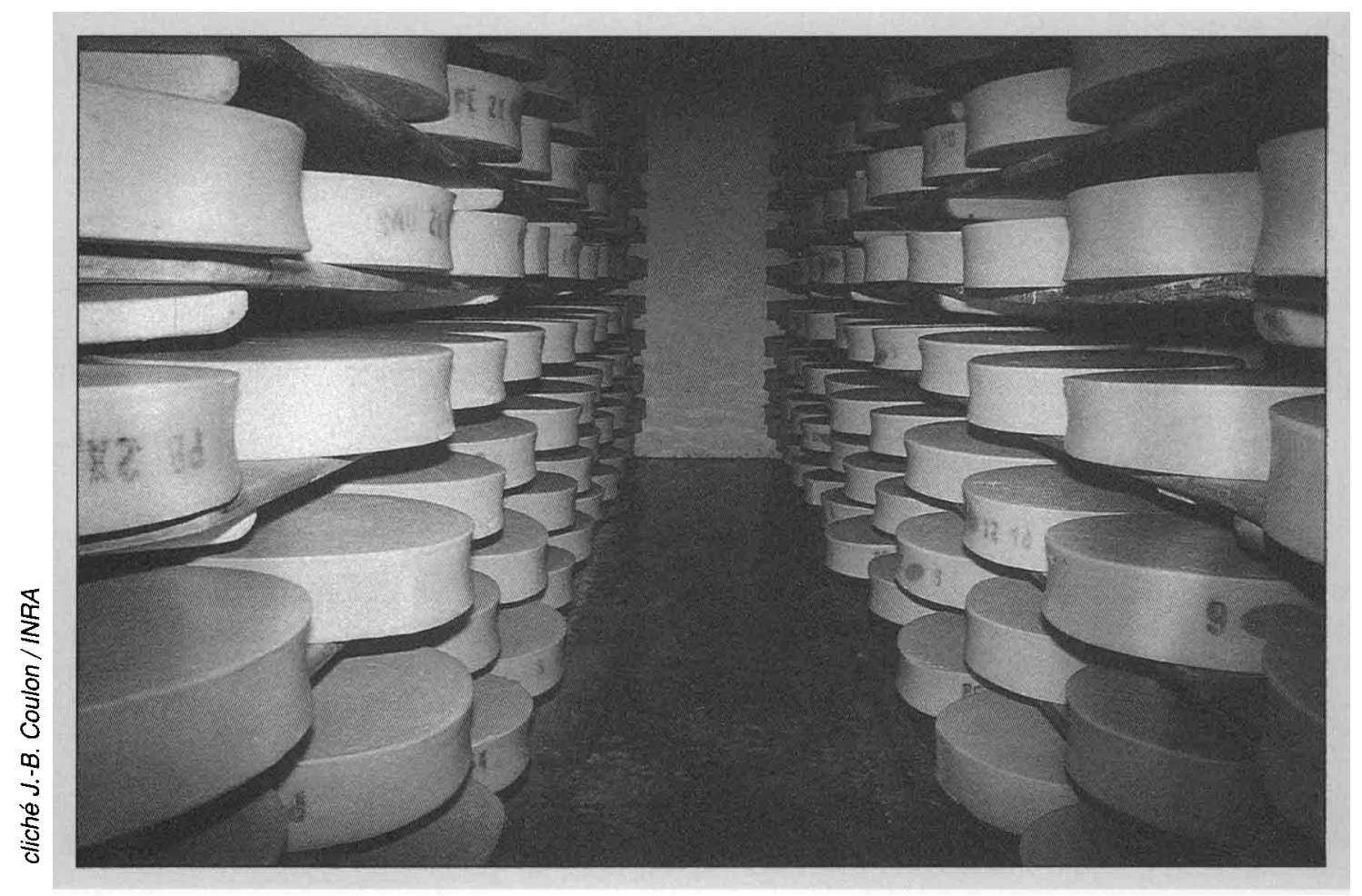

pertinentes et au total plus efficaces, face à la diversité de leurs livreurs.

\section{Conclusion}

Cette rapide revue des manières d'aborder le concept de qualité et de ses principales difficultés d'emploi fait ressortir les éléments suivants :

- La mise en place d'une véritable politique de qualité relève d'une démarche collective à l'échelle de la filière, à l'intérieur de laquelle cœxistent généralement, selon les participants, des conceptions et des stratégies très diverses en matière de qualité. Ceci résulte à la fois de la diversité de ces acteurs et des différentes formes de coordination pouvant exister entre eux. La principale difficulté consiste donc à identifier et négocier collectivement un concept de qualité qui puisse être partagé par tous, et traverser l'ensemble de la filière. Il semble important, en particulier, que les producteurs agricoles comme les entreprises de transformation aient une claire perception du positionnement du produit final sur le marché, et donc une bonne compréhension de la demande de qualité. De plus, la motivation et la mobilisation de l'ensemble du personnel de l'entreprise apparaissent comme des éléments essentiels d'une politique de qualité.

- Tous les acteurs d'une filière interviennent, directement ou indirectement, à différents niveaux du processus d'élaboration de la qualité d'un produit (mode de définition, évaluation, élaboration proprement dite). Cette qualité résulte d'un processus complexe, dans lequel les relations entre agents sont déterminantes. Une voie possible, pour aborder ce processus dans sa complexité, est celle de la modélisation systémique. Le concept actuel de qualité se développe en effet, de manière plus ou moins explicite, à partir d'une perception très systémique du fonctionnement des filières, depuis la production jusqu'à la mise 
en marché. Il en ressort que l'instruction de ce type de dossier relève désormais de démarches pluridisciplinaires intégrées associant chercheurs et praticiens dans le cadre d'un partenariat d'un nouveau type et d'une démarche de recherche-action (Avenier 1989).

Travail réalisé dans le cadre du programme Recherche-Développement des Alpes du Nord (GIS, 1 rue du Château, 73000 Chambéry).

\section{Remerciements}

L'auteur remercie Geneviève Baud, Annie Soyeux et Egizio Valceschini, pour leur aide déterminante dans la recherche des documents qu'elle a utilisés et leurs conseils constructifs, ainsi que Jean-Baptiste Coulon, Gérard Larrieu et Emmanuel Mingasson, pour leur lecture critique. Merci enfin à Etienne Landais, pour l'appui qu'il a fourni à la conception et à la rédaction de ce texte.

\section{Références bibliographiques}

AFNOR, 1982. Gestion de la qualité. Vocabulaire. Norme expérimentale X 50.109 .

AVENIER M.J., 1989. "Méthodes de terrain" et recherche en management stratégique. Economies et Sociétés, 14 : 199-218.

BOISARD P., LETABLIER M.T., 1986. Liens locaux de production et standards industriels. Le cas du Camembert. Cahiers du Centre d'Etudes de l'Emploi, vol.15, 146 pages. Paris, PUF.

BOURDIEU P., 1979. La Distinction : critique sociale du jugement. Paris, Ed. de Minuit, 670 pages.

BROSSIER J., VALCESCHINI E.(éd.), 1991. Les exploitations et leur environnement. INRA, Paris, 329 pages.

BUISSON M., GUGLIELMI M., 1990. Qui perd et qui gagne au jeu des quotas laitiers. Une approche à partir des travaux conduits en Rhône-Alpes. Economie rurale, $199: 14-18$

BURON I., 1990. La qualité et les nouveaux produits alimentaires. Revue des IAA, $3: 799-804$.

CANEILL J., CAPILLON A., 1990. Gestion de la qualité des productions végétales au plan régional. Economie rurale, 198, p 23.

COULON JB., ROYBIN D., CRISTOFINI B., 1990. Production laitière et fonctionnement des exploitations: facteurs de variations dans les exploitations du Pays de Thônes (Haute-Savoie). INRA Prod. Anim., 3 : 287- 298.

CREYSSEL P. et coll., 1991. l'Agro-alimentaire. Pour une stratégie de normalisation. Enjeux, 113, 88 pages.

DEMEYER D., 1991. Production et qualité de la viande. Communication au Colloque international de l'Agriculture-Environnement-Qualité (Nancy, 12-13 septembre 1991), ENSAIA/INPL- Nancy.

EYMARD-DUVERNEY F.,1989. Conventions de qualité et formes de coordination. Revue économique, $2: 329-359$.

GILIBERT J., BAUDRY J., BOUDINEAU J.M, DENIS D., 1987. Les producteurs de lait d'une filière fromagère AOC. Rapport CEE/INRA-SAD, Unité de Recherche sur les Systèmes Herbagers Normands, 112 pages.

GRIGNON C. et GRIGNON CH., 1980. Styles d'alimentation et goûts populaires. Revue française de sociologie, Vol.XXI (4) : 531-569.

HERPIN N., 1980. Comportements alimentaires et contraintes de temps. Revue française de sociologie, Vol.XXI (4) : 599-628.
JURAN J.M., 1974. Quality control hand book. NewYork, Mc Graw Hill Book Company.

LANDAIS E., 1987. Recherches sur les systèmes d'élevage. Questions et perspectives. Versailles, INRA, Document de travail de l'URSAD Versailles-DijonMirecourt, INRA-SAD Versailles, $73 \mathrm{p}$.

LEBAIL M., VALCESCHINI E., 1990. Qualité des produits agricoles et coordination dans les filières agro-alimentaires. Economie rurale, 198, p. 41.

MULTON JL, 1985. La qualité des produits alimentaires. Paris, Lavoisier, Collection Sciences et Techniques Agroalimentaires, TEC et DOC. APRIA. 487 pages.

PERRIER-CORNET PH., 1990. Les filières régionales de qualité dans l'agro-alimentaire. Etude comparative dans le secteur laitier en Franche-Comté, Emilie Romagne, Auvergne. Economie Rurale, $195: 27-33$.

RICHARD A., 1990. Fromages, horizon 2000. Revue "Linéaires", $43: 34-36$.

SYLVANDER B., 1991. Conventions de qualité et marchés des produits de qualité spécifique dans l'agro-alimentaire. Document de travail. INRA Economie, Toulouse, 28 pages.

SYLVANDER B., MOUGIN L., 1986. La consommation des produits biologiques à Toulouse. Toulouse, INRA Economie, 210 pages.

THIEBAUT L., 1991. Qualités des produits agricoles et qualités de l'environnement : des synergies limitées. Communication au Colloque international AgricultureEnvironnement-Qualité (Nancy, 12-13 septembre 1991). ENSAIA/INPL- NANCY, 9 pages.

TOURAILLE C, KOPP J., VALIN C., LECLERQ B., 1981. Qualité du poulet. I: Influence de l'âge et de la vitesse de croissance sur les caractéristiques physico-chimiques et organoleptiques de la viande. Arch. Geflügelk., $45: 69-76$.

VALCESCHINI E., HEINTZ W., 1991. La gestion de l'incertitude sur la qualité des blés par les organismes collecteurs et stockeurs. Une approche par les formes de coordination. In Brossier et Valcheschini (ed.). "Les exploitations et leur environnement", INRA Paris, 1991. 11 pages.

VALCESCHINI E., 1991. Qualité des produits agricoles et formes de coordination entre exploitations agricoles et firmes agro-alimentaires. Le cas du secteur des légumes de conserve. Les institutions dans le secteur des légumes de conserve. Les transformations de "l'économie contractuelle". CNRS-PIRTTEM/MRT-TET/INRA-ESR, INRASAD-Paris-Grignon, 39 pages.

\section{Summary}

Reflexions on the concept of quality of agricultural products. The example of milk for cheese in french North Alps.

The concept of quality leads to different interpretations. Agricultural product quality is not mechanically controlled by demand, but is the result of a complex process, in which relationships between producers and firms are very important. To set up a quality strategy a collective mobilization of these agents is required. A modelling approach is proposed for the construction of decision making aids that will enable economic actors to better control the quality of their products.

DUBEUF Brigitte, 1992. Réflexions sur le concept de qualité dans l'agro-alimentaire. L'exemple de la filière lait fromage dans les Alpes du Nord. INRA Prod. Anim., 5 (2), $91-101$ 